\title{
Towards Understanding of Influence of Restricted Geometry on Self-Diffusion in Porous Media
}

\author{
I. STANKOVIC* \\ Scientific Computing Laboratory, Institute of Physics, Belgrade, Serbia
}

\begin{abstract}
Novel simulation procedure of self-diffusion through model porous media is presented. The procedure is based on extensive use of molecular dynamic method. The introduced diffusion simulations have a potential to become a valuable design and development tool applications involving transport in porous media, e.g. filters or porous sensors. The porous membranes are created using phase-separating model with high surface tension and its properties are characterized and compared with existing porosimetry measurements and small angle scattering experiments. These membranes are then filled with a gas of diffusing particles. Relations between transport coefficients and material structure are discussed.
\end{abstract}

PACS numbers: 82.56.Lz, 82.33.Ln, 83.10.Mj, 82.75.Fq

\section{Introduction}

The physics of interaction of diffusing particles with porous media has attracted a lot of attention, due to its significance in major technological areas such as selective catalysis, electrochemical sensors and filters. With powerful measurement techniques like ellipsometry and small angle scattering [1, 2], one can obtain pore surface size and distribution information for thin films obtained by coating industry using sol-gel method [3]. In addition titanium- $\left(\mathrm{TiO}_{2}\right)$ and cerium-dioxide $\left(\mathrm{CeO}_{2}\right)$, obtained with the sol-gel method, are increasingly used as functional membranes due to their dual catalytic and structural properties. One of the advantages of sol-gel method is that various properties of porous media can be obtained by variation of the process parameters. This fact provides us with opportunity to develop industrial processes in which porous materials with optimal pore geometry characteristics are produced for various applications [4]. On the other hand, there is still no agreement which microscopic geometrical measures and structural properties of the pores are needed to completely characterize transport through porous media in many applications. In this work, we demonstrate a simulation procedure which enables us to create porous media by mimicking the sol-gel process and then we demonstrate use of the obtained porous media for simulations of diffusion of gases interacting with short-range attractive or repulsive forces.

The simulation procedure in this work consists of two steps. In the first step, porous media are created using phase separation simulations. The evolution of phase

\footnotetext{
* e-mail: igor.stankovic@phy.bg.ac.rs
}

separation can be stopped at different time instances which results in family of porous structures with continuously varying structural characteristics. In the second step, the pores of obtained porous media are filled with a gas of diffusing particles.

\section{Porous media creation}

We are applying a coarse-grained approach. In a coarse-grained model for the sol-gel process, each nanoparticle is replaced with a single "gel particle" while interaction model is accounting for particle interactions. The used model potential is the sum of two contributions to the total potential energy $E$ : a conventional binary interaction term through a two-body interaction potential $U$ and a term stemming from a density dependent functional $F$, see Ref. [5],

$$
E=\sum_{i=1}^{N}\left[F\left(\rho_{i}\right)+\sum_{j>i}^{N} U\left(r^{i j}\right)\right],
$$

where $N$ is number of atoms at positions $\boldsymbol{r}_{i}=1,2, \ldots$, $N$ and $r_{i j}$ is the norm of the relative vector $\boldsymbol{r}_{i j}=\boldsymbol{r}_{i}-\boldsymbol{r}_{j}$ between atoms $i$ and $j$.

The particle-particle interaction will be relatively short-ranged and of a Lennard-Jones (LJ) type in neutral solution. This approximates particle-particle interactions by the van der Waals force between atomic surfaces. We applied here a radially symmetric short ranged attractive potential (SHRAT) form

$$
\begin{aligned}
& U(r)=\phi_{0} r_{0}^{-4}\left[3\left(r_{\text {cut }}-r\right)^{4}-4\left(r_{\text {cut }}-r_{\text {min }}\right)\left(r_{\text {cut }}-r\right)^{3}\right], \\
& r \leq r_{\text {cut }}
\end{aligned}
$$

and $U(r)=0$ otherwise, with an energy scale $\phi_{0}$, a length scale $r_{0}$, an interaction range $r_{\min }$, and a smooth cut-off 
radius $r_{\text {cut }}$. A many-body term is added in order to increase surface tension. As it will be demonstrated in this latter section, the resulting porous walls are more connected and qualitatively closer to that seen in experiment compared to the ones obtained with purely binary interactions, cf $[3,6]$. The many-body potential is also given in polynomial form

$$
F(\rho)=\phi_{0} \sum_{k=2,4 \ldots} F_{k}\left[\left(\rho-\rho_{\mathrm{des}}\right)^{k}-\left(w_{0}-\rho_{\mathrm{des}}\right)^{k}\right] r_{0}^{3 k},
$$

where $\rho_{\text {des }}$ is the desired embedding number density and $F_{k}$ are embedding strengths, being part of the model. The local embedding density $\rho_{i}$ is constructed from the radial coordinates of surrounding atoms and requires the choice of a weighting function $w(r), \rho_{i}=$ $\sum_{j \neq i} w\left(r_{i j}\right)+w(0)$. We use the normalized Lucy [7] weight function: $w(r)=w_{0}\left(1+3 r / r_{\text {cut }}\right)\left(1-r / r_{\text {cut }}\right)$, $r \leq r_{\text {cut }}$ with $w_{0}=w(0)=105 /\left(16 \pi r_{\text {cut }}\right)$. In this work, we used $F_{0}=1$ and $F_{k}=0$. For $k>0, r_{\min }=2^{1 / 6}$ and $r_{\text {cut }}=1.6$ are fixed.

The main feature of the model is a controlled mismatch between preferred local and the global number densities. In order to obtain porous structures we choose initial configuration with (bulk, cell wall) density $\rho_{\text {des }}=1$ (reduced units) larger than the overall number density $n \equiv N / V=0.3$. Initially particles inside simulation cell are homogeneously distributed. After simulation onset, holes surrounded by metal are formed which keep with well connected and stable walls, cf. Ref. [6]. This is caused by high surface tension. Figure 1 shows formation of model porous media. The series of three pictures illustrates the continually evolving structure resulting from implemented particle potentials, which is qualitatively different from previously seen behavior of LJ fluid.

To compare NEMD simulation results with experimental data, we relate constitutive properties of used model with experimental data. Any measurable quantity $Q$ with a dimension $[Q]$ specified in SI units of $\mathrm{kg}$, $\mathrm{m}$ and $\mathrm{s}$ is converted into dimensionless reduced LJ units by a reference quantity $Q_{\text {ref }}=m^{\alpha+\gamma / 2} r_{0}^{\beta+\gamma} \Phi_{0}^{-\gamma / 2}$ for $[Q]=\mathrm{kg}^{\alpha} \mathrm{m}^{\beta} \mathrm{s}^{\gamma}$ such that quantities $m, r_{0}$ and $\Phi_{0}$ provide the scales via the interaction potential and the equations of motion. In this work we choose $T_{\text {ref }}=40 \mathrm{kK}$, $m=0.53 \times 10^{-18} \mathrm{~kg}$, and $r_{0}=50 \mathrm{~nm}$ for $\mathrm{TiO}_{2}$ nanoparticles based on previous considerations, cf. Ref. [5].

Two methods are used in this work to characterize obtained model porous media. Both methods provide us with quantities measurable in experiments:

(1) numerical integration using Monte Carlo methods is used to calculate surface and volume of dense phase, i.e. pore walls. To estimate the volume, a sequence of random points is generated and tested if they fall within dense phase. The volume occupied by pore walls is then estimated as this fraction multiplied by total volume. Surface of the pores is calculated as derivative of volume with respect to particle radius.

(2) For sufficiently large systems, with more than 10000 particles, it is possible to perform spatial averag-

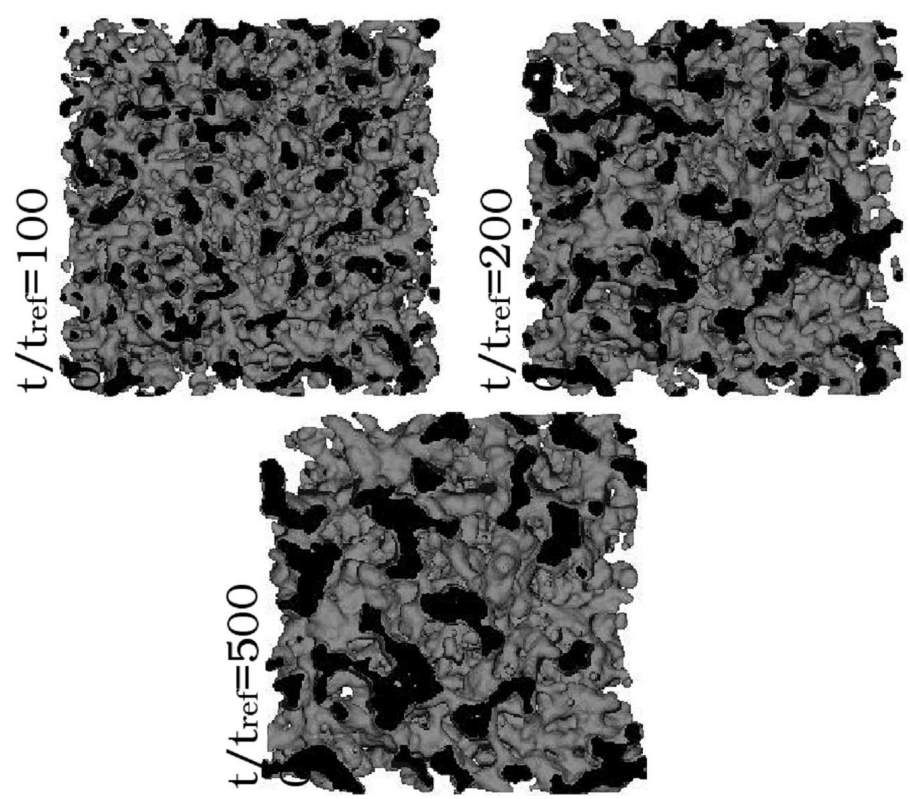

Fig. 1. Snapshots of temporal evolution of structure in a system of 50000 particles. Snapshots were taken at different times in reduced LJ units.
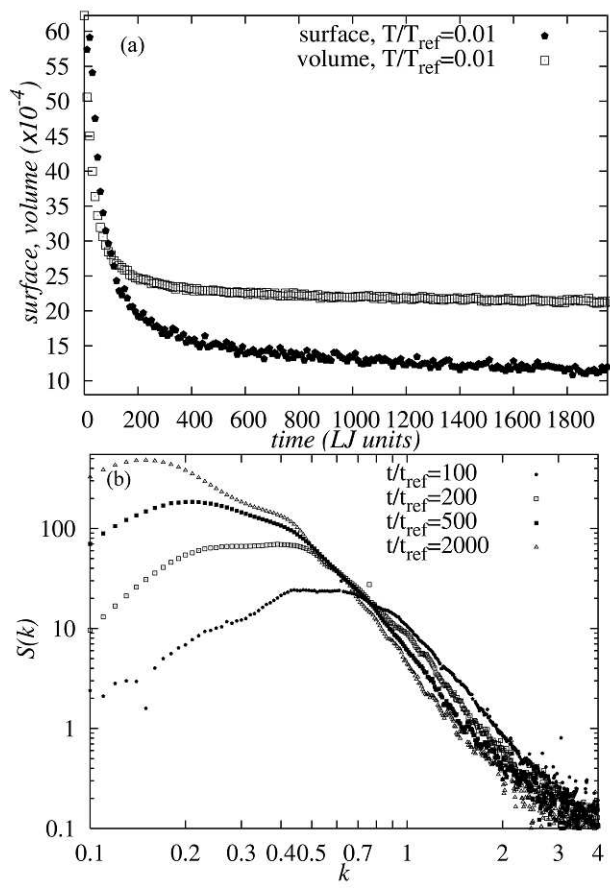

Fig. 2. (a) Evolution of surface and volume size in reduced (LJ) units with time. (b) Structure factor dependence on wave number is shown in $\log -\log$ scale. Wave vector $k=1$ in reduced units corresponds to wavelength of $0.3 \mu \mathrm{m}$ in $\mathrm{TiO}_{2}$. 
ing and calculate structure factor $S(k)$, which is related to pair distribution $g(r)$, by the Fourier transformation

$$
S(k)=1+4 \pi n \int_{0}^{\infty}[g(r)-1] r^{2} \frac{\sin (k r)}{k r} \mathrm{~d} r .
$$

Using structure factor we can quantify self-similarity of the structure over in a range of different scales at micrometer level using fractal surface dimension $D_{\mathrm{s}}[3]$. A value of the fractal dimension of the aggregating system can be obtained from high wave vector side of the small angle scattering peak through relation $S(k) \propto$ $k^{-\left(6-D_{\mathrm{s}}\right)}$.

The rate of structure coarsening is very fast at onset of simulation and slows down with the time, i.e., curves in Fig. 2a are becoming rapidly flatter with time. This can be explained by increasing size of pores walls which makes them more stable. After $t=200$, surface size still decreases while volume is stable. This is caused by surface getting more flat. Surface dimension of created porous media is 2.4 which is comparable to many silica aerogels, $c f$. Fig. $2 \mathrm{~b}$ and Ref. [3]. Flattening of the porous media surface can be also followed in Fig. $2 \mathrm{~b}$ as shortening of the linear region with the time.

\section{Simulations of self-diffusion in porous media}

The sponge is filled with particles interacting with each other through SHRAT potential, and interaction between wall and gas particles is repulsive with smooth cut-off at $r_{\min }$ :

$$
U_{\text {rep }}(r)=\phi_{0} r_{0}^{-4}\left(r_{\min }-r\right)^{4} \text {. }
$$

$r \leq r_{\min }$ and $\Phi(r)=0$ otherwise. Gas is thermostated indirectly through the collisions with the sponge wall particles. Temperature of the particles at the sponge surface is controlled by rescaling of their velocities, while positions of particles inside of the sponge are fixed to stabilize the structure and prevent a change of its porosity, $c f$. Fig. 3.

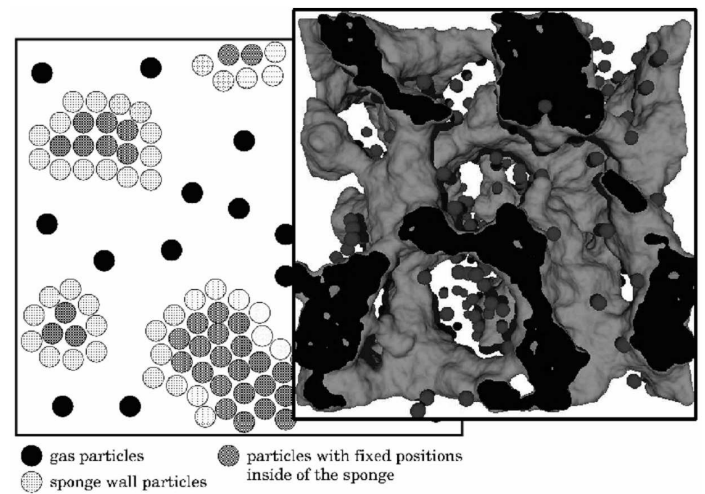

Fig. 3. Scheme of the diffusion transport simulations (left) and part of the system (right). Position of particles inside of the porous walls are fixed, thermostat is applied for controlling temperature of particles on the surface.

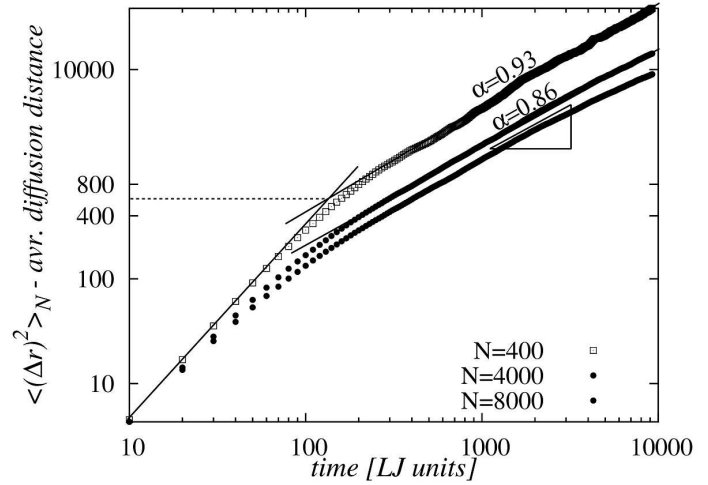

Fig. 4. Mean square displacement (average diffusion distance) of SHRAT particle gas inside the GEAM matrix at temperature $T / T_{\text {ref }}=0.01$, for $N=400,4000$, 8000 SHRAT gas particles.

In Fig. 4 molecular dynamics results are given at temperature $T / T_{\text {ref }}=0.01$ for dilute SHRAT gas with 400 , 4000, and 8000 particles. Collision (free flight) time, $t_{\text {coll }} \approx 110$ for 400 particles, is determined by typical pore diameter $R_{\text {coll }} \approx 24$. For 4000 particles, collision time is shorter, $t_{\text {coll }} \approx 40$, due to the SHRAT-SHRAT particle collisions. We observe clusters of SHRAT particles formed during the simulation. For this reason at times larger than collision time, the mean square displacement increases with curvatures $\alpha=0.86,0.93,\left\langle\Delta r^{2}\right\rangle \propto t^{\alpha}$, for systems with 400, 4000 particles, respectively. In case of normal diffusion, one would expect $\alpha=1$, according to the Einstein relation, $\left\langle\Delta r^{2}\right\rangle=6 D t$. Sub-diffusion of agglomerating particles could have important influence on particulate filters operation mechanisms [8]. In particulate filters soot (carbon) nanoparticles are collected from exhaust gases and burned in pores. Previous studies showed that there is an influence of agglomeration on soot burning chemistry, see Ref. [9]. If proven, sub-diffusion in particulate filters will mean a significantly larger accumulation of soot particles at entrance into porous media, as compared to normal diffusion model.

\section{Conclusion}

In this work, a modeling approach is introduced with special attention to reproducing porous media and characterizing it in similar way like in experiment. A simple and computationally efficient procedure is introduced for creation of realistic porous media. Already in early stage of application of these simulations, it was demonstrated that sub-diffusion could be studied. The presented simulations have a potential to become a valuable research tool for creation of models connecting a limited number of measurable microstructural properties and transport characteristics of the porous media. Such models would be an important contribution to creation of reliable and time efficient 3D models linking experimental measurements and transport properties. 


\section{Acknowledgments}

This work was supported by the Serbian Ministry of Science and Technological Development under project No. OI141035 and the European Commission under EU Centre of Excellence grant CX-CMCS.

\section{References}

[1] V. Rouessac, R. Coustel, F. Bosc, J. Durand, A. Ayral, Thin Solid Films 495, 232 (2006).

[2] R. Vacher, T. Woignier, J. Pelous, Phys. Rev. B 37, 6500 (1988).

[3] A. Golubović, M. Šćepanović, A. Kremenović, S. Aškrabić, V. Berec, Z. Dohčević-Mitrović, Z.V. Popović, J. Sol-Gel Sci. Technol. 49, 311 (2009).
[4] H. Hayashi, RधD Rev. Toyota Central Research Development Laboratory 38, 17 (2003).

[5] I. Stankovic, S. Hess, M. Kroger, Phys. Rev. E 70, 066139 (2004).

[6] J. Lodge, D. Heyes, J. Chem. Phys. 109, 7567 (1998).

[7] L. Lucy, Astron. J. 82, 1013 (1977).

[8] H. Yanagihara, W. Brandstätter, N. Ohashi, B. Gschaider, J. Leixnering, I. Stankovic, to appear in Topics in Catalysis.

[9] J. Bhatta, R. Lindstedt, Proc. Combust. Inst. 32, 713 (2009). 